\title{
Un método empírico para el proporcionamiento de mezclas de Morteros de Cemento Portland para Albañilería
}

\author{
Ing. ALEJANDRO SALAZAR \\ Prof. de la Universidad del Valle - Sección de Materiales \\ Cali-Colombia
}

\section{$R E S U M E N$}

En el medio colombiano, dado el auge de la aplicación de sistemas constructivos no tradicionales y acogiéndose al nuevo Código de Construcciones Sismicas, se necesita trabajar con morteros de cemento de carácteristicas definidas y reproducibles.

Se realizó un análisis de las variables que inciden en el comportamiento de los morteros y se formuló una experimentación que permitiera comprobar y correlaccionar la interdependencia entre éstas.

Con mamposteros profesionales, se estableció el rango de fluidez apropiado y se comprobó el efecto del retemplado en las propiedades resistentes de los morteros.

Se presenta un método de dosificación que satisface óptimamente los aspectos técnicos como económicos y a su vez considera los métodos tradicionales de trabajo de nuestro obrero de construcción.

\begin{abstract}
$S U M M A R Y$
In Colombian ways and means, since the application of no traditional constructive systems is growing up, and having recourse to the new Seismic Construction Code, it is necessary to work with cement mortars of defined and reproducible characteristics.
\end{abstract}

An analysis of the variability affecting the mortars behaviour was carried out, and an experimentation which would allow to test and to correlate the interdependence between them, was expressed.

With professional stonemasons, the suitable fluidity rank was established, and the effect of the retempered on the resistance properties of the mortars, was tested.

A method of dosage is presented, which best satisfies the technical and economic aspects and considers the traditional work methods of our construction worker.

\section{INTRODUCCION}

\subsection{Justificación $y$ antecedentes}

En el medio de construcción regional y aún a nivel nacional, no se emplea un método racional que permita definir una mezcla de un mortero tal que satisfaga los requerimientos técnicos de la obra, dentro de rangos tolerables y utilizando los materiales locales.

Con el auge en el empleo de la mamposteria estructural asi como de diversos elementos prefabricados que requieren de un acople y terminado adecuado, se están requiriendo morteros de cualidades y calidades particulares como son: resistencias mecánicas definidas, fluidez (trabajabilidad) especifica, impermeabilidad y capacidad de retención de agua altas, dureza superficial apropiada y aún más alta durabilidad frente al ambiente. Todo lo anterior y aun otras caracteristicas más, no se consiguen sin un método de proporcionamiento adecuado amén de un óptimo mezclado, transporte y colocación (3). 
La evolución de los sistemas constructivos tendentes a cambiar la tradicional tecnologia de construcción en Colombia, comienza en la primera parte de la década de los 70 y se acentúa notablemente en su segunda mitad, introduciéndose obviamente conceptos que inciden no sólo en dichas técnicas constructivas sino en las características de los materiales compuestos requeridos, tales como el hormigón y los morteros de albañileria (4).

Dada la carencia de información al respecto, se ha venido trabajando, en el mejor de los casos, con normas y especificaciones de otros países cuyo inconveniente de uso radica en estar formuladas para el empleo de materiales cuyas características físico-químico-mecánicas difieren de los utilizados en el medio. Dichos métodos no proporcionan resultados óptimos y engendran con su aplicación conceptos contradictorios y empirismos respaldados por una "Experiencia" no cuestionada ni cuestionable.

El Instituto Colombiano de Normas Técnicas (ICONTEC) produce en 1977 la norma C 4.99/77 que establece los requisitos de los morteros para usos en estructuras de Mampostería (5). La norma mencionada es una traducción literal de su equivalente a la ASTM C-270-73. Han existido algunos intentos de cambiar la aplicación de esta norma del ICONTEC e incluso se ha propuesto un nuevo proyecto de norma (6), aunque éste no se encuentra respaldado por un trabajo de investigación exhaustivo al respecto (7).

En 1982, la sección de Materiales de la Universidad del Valle se formula un tema de investigación sobre esta temática a la par que desarrolla un método de diseño de mezclas para hormigones fluidos (2) (10).

Conscientes de haber iniciado el recorrido por un camino aún largo, con el presente trabajo de investigación y sus resultados se espera contribuir al enriquecimiento de la discusión sobre el tema y a aportar algunas soluciones específicas.

\subsection{Objetivos}

Se pretende definir un método de cálculo para dosificar morteros de albañilería.

En el estudio se abarcan los siguientes aspectos:

- Caracterización fisica de los materiales que integran el mortero.

- Estudio de las propiedades fisicas del mortero: trabajabilidad, fluidez, retemplado, rendimiento, peso volumétrico, etc...

- Caracteristicas mecánico-resistentes de los morteros, en función de:

a) La variación de la dosificación para una relación agua/cemento constante.

b) La variación de la relación agua/cemento para una dosificación constante.

c) La variación de la relación agua/cemento para una fluidez constante.

d) La variación de la dosificación para un flujo constante.

e) La adición de agua de retemplado para recuperar la fluidez inicial a tiempos definidos.

Con lo anterior se establecerá:

f) La fluidez que determina la trabajabilidad óptima.

g) Las curvas de hinchamiento y pesos unitarios de la arena en función del contenido de humedad que permitirá relacionar las dosificaciones por peso y por volumen. 
h) La correlación entre la resistencia mecánica a compresión y: la relación agua/cemento y la relación arena/cemento.

i) El peso volumétrico, suelto y compactado, de los morteros de distintas dosificaciones.

j) Con los resultados de (h) e (i) se planteará el método de diseño.

k) Las curvas de retemplado que simularán las condiciones de trabajo en obra y permitirán determinar la influencia de la readición de agua en la resistencia mecánica de los morteros.

\section{METODOLOGIA}

Se puede resumir así (8):

\subsection{Recolección, Almacenamiento, Clasificación y Homogenización de las Materias Primas}

Se trabajó con los materiales más utilizados en la región de Cali, procurando un muestreo representativo y un manejo adecuado de ellos que garantizara la mayor reproducibilidad del método encontrado.

\subsection{Caracterización de los Materiales}

Se emplearon las Normas ICONTEC pertinentes:

Para el cemento se realizaron los ensayos correspondientes a las normas de:

- Superficie especifica. ICONTEC-33.

- Residuo sobre tamiz $\neq(74)$. ICONTEC-226.

- Gravedad especifica. ICONTEC-221.

- Consistencia normal. ICONTEC-110.

- Tiempo de fraguado. ICONTEC-118.

- Resistencia mecánica a compresión. ICONTEC-220.

Para la arena se hicieron ensayos correspondientes a las normas de:

- Granulometria. ICONTEC-77.

- Pesos unitarios. ICONTEC-92.

- Peso específico y absorción. ICONTEC-237.

- Impurezas orgánicas. ICONTEC-127.

— \% de hinchamiento: se usó el procedimiento descrito en (9). 


\subsection{La Dosificación de los Morteros}

Cada dosificación por peso se planteó en superficie seca saturada. Con base en los porcentajes de humedad total y absorción de la arena se efectuaron las correcciones pertinentes.

Se trabajó con dosificaciones entre $1: 2(1$ parte de cemento $: 2$ partes de arena) hasta $1: 4$ y se tomaron cubos según norma ICONTEC 220. La relación agua/cemento se varió para cada una de las dosificaciones definidas, determinándose para cada variación la fluidez (Norma ICONTEC111) y las resistencias mecánicas a compresión a 3,7 y 28 dias).

Después de varias consultas y ensayos con diferentes mamposteros experimentados, estableció un rango de fluidez entre: 95 y $110 \%$.

Se hicieron dosificaciones en volumen, desde $1: 2,5$ hasta $1: 3,5$, donde para cada una de ellas se varió la relación agua/cemento en función del flujo y se deteminaron las resistencias mecánicas a compresión a las edades mencionadas anteriormente.

\subsection{Determinación del peso volumétrico suelto y compactado del mortero fresco}

Se empleó el mismo método que se establece para los áridos en la norma ICONTEC-92, utilizándose el recipiente recomendado para las arenas. Se hizo para todos los morteros preparados cuya fluidez entraba en el rango definido anteriormente.

\subsection{El proceso de retemplado}

Consiste en la adición de agua a un mortero preparado que ha perdido fluidez, con el fin de recuperar ésta y poderlo trabajar. Como es una práctica común, se propuso averiguar su efecto sobre la calidad resistente de los morteros. Mediante pruebas de fluidez se encontró, que bajo condiciones de obra, era notable la pérdida de fluidez después de transcurrir 45 minutos de preparada la mezcla. Se decidió entonces retemplarla a este tiempo; como a los 90 minutos había perdido la trabajabilidad nuevamente se volvió a retemplar e igual ocurrió y se hizo a los 135 minutos. En cada oportunidad, después del retemplado, se tomaron muestras para ensayos de resistencia a compresión. Así mismo, se efectuó un retemplado a los 135 minutos después del mezclado inicial.

Para establecer comparaciones del efecto del retemplado, se tomaron muestras a los 45, 90 y 135 minutos de mezclas sin retemplar, que fueron ensayadas para establecer las resistencias mecánicas a compresión. Se siguió este procedimiento de retemplado a todas las dosificaciones planteadas.

\subsection{El contenido de humedad del mortero fresco}

Se decidió realizar para cada dosificación, en el rango de fluidez definido, una medida de la humedad total efectuando un secado en estufa a $110^{\circ} \mathrm{C}$. Se desea conocer si hay una humedad caracteristica que define a un mortero, independiente de su dosificación.

\section{CARACTERISTICAS DE LOS MATERIALES}

\subsection{Arenas}

En la tabla 1 y el gráfico 1 se presentan las caracteristicas de la arena empleada: 
TABLA 1

Características de la arena

\begin{tabular}{|c|c|c|}
\hline Caracteristicas & Arena Natural & Arena Clasificada \\
\hline - Granulometria & (\% pasa) & (\% pasa) \\
- Tamiz: 3/8" $(9.51 \mathrm{~mm})$ & 100.0 & 100.0 \\
$\neq 4(4.76 \mathrm{~mm})$ & 97.6 & 96.9 \\
$8(2.38 \mathrm{~mm})$ & 94.6 & 84.9 \\
$16(1.19 \mathrm{~mm})$ & 82.9 & 46.4 \\
$30(595 \mu)$ & 45.3 & 13.6 \\
$50(297 \mu)$ & 13.3 & 1.9 \\
$100(149 \mu)$ & 1.8 & \\
\hline
\end{tabular}

- Pesos Unitarios: $\left(\mathrm{kg} / \mathrm{m}^{3}\right)$

$\begin{array}{llc}\text { - Suelto } & 1.579 & 1.290 \\ \text { - Compactado } & 1.691 & - \\ \text { Absorción (\%) } & 2.50 & 2.48 \\ \text { Gravedad específica (SSS) } & 2.62 & 2.58 \\ \text { Módulo de finura } & 2.77 & 2.56\end{array}$

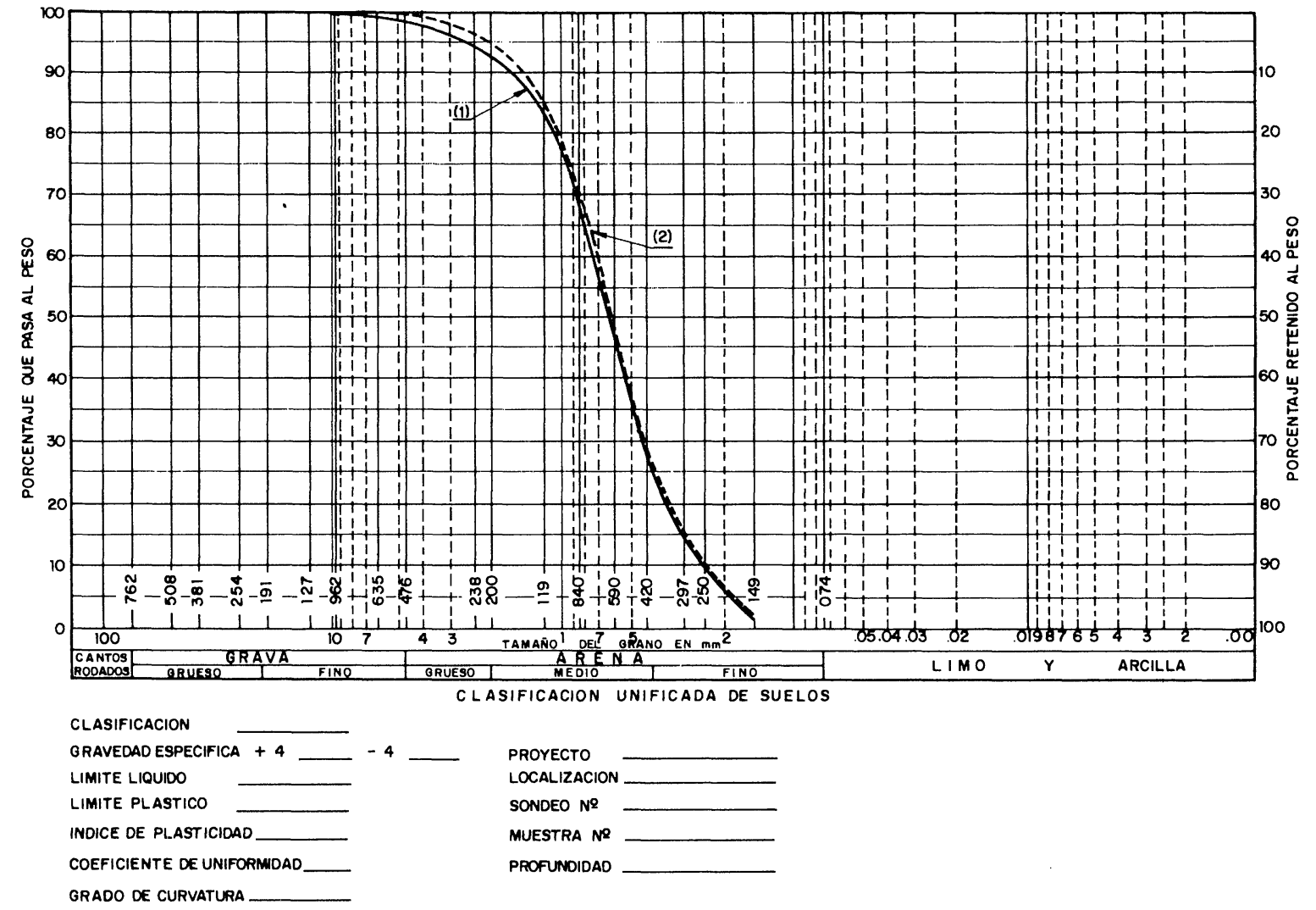

Gráfico 1 


\subsection{Cemento}

Corresponde al material producido y empleado en la región. Sus características son:

- Superficie especifica Blaine $\left(\mathrm{cm}^{2} / \mathrm{g}\right)$ :

- Residuo en tamiz $\neq 200(74 \mu)$. (\%)

- Gravedad específica

- Consistencia normal (\%)

- Tiempo de fraguado (minutos)

75

- Resistencias a compresión $\left(\mathrm{kg} / \mathrm{cm}^{2}\right)$ :

- 3 días

- 5 días

- 7 días

- 28 días

\subsection{Agua}

Corresponde a la suministrada para consumo humano por las empresas Municipales de Cali.

\section{ANALISIS DE RESULTADOS (8)}

\subsection{Variación de los Pesos Unitarios de las Arenas del Rio Cauca, en función del contenido de humedad}

En base a los resultados conseguidos, se hizo un procesamiento matemático con el fin de establecer una correlación estadística de ellos. Se definió una correlación lineal que se expresa a continuación:

a) En el rango de humedad entre 0.0 y $7.5 \%$ se obtuvo la siguiente expresión:

$$
\mathrm{Y}=1286,9-40,3 \mathrm{X}(1)
$$

Coeficiente de Correlación $(r)=0.93$

$$
\begin{aligned}
& \mathrm{X}=\% \text { de humedad de la arena } \\
& \mathrm{Y}=\text { Peso Unitario Suelto }\left(\mathrm{kg} / \mathrm{m}^{3}\right)
\end{aligned}
$$

b) En el rango de humedad entre 7.5 y $26.0 \%$ se obtuvo la siguiente expresión:

$$
\mathrm{Y}=910+23.3 \mathrm{X}(2)
$$

Coeficiente de Correlacion $(\mathrm{r})=0.98$

$$
\begin{aligned}
& \mathrm{X}=\% \text { de humedad de la arena } \\
& \mathrm{Y}=\text { Peso Unitario Suelto }\left(\mathrm{kg} / \mathrm{m}^{3}\right)
\end{aligned}
$$

En el gráfico 2 se observa que hay un mimimo de peso unitario determinado por la intersección de las dos rectas. El incremento de humedad hasta un valor de $7.5 \%$ genera un decrecimiento 
en el Peso Unitario de la arena; posterior a esta cifra aparecerá un incremento del Peso Unitario mencionado.

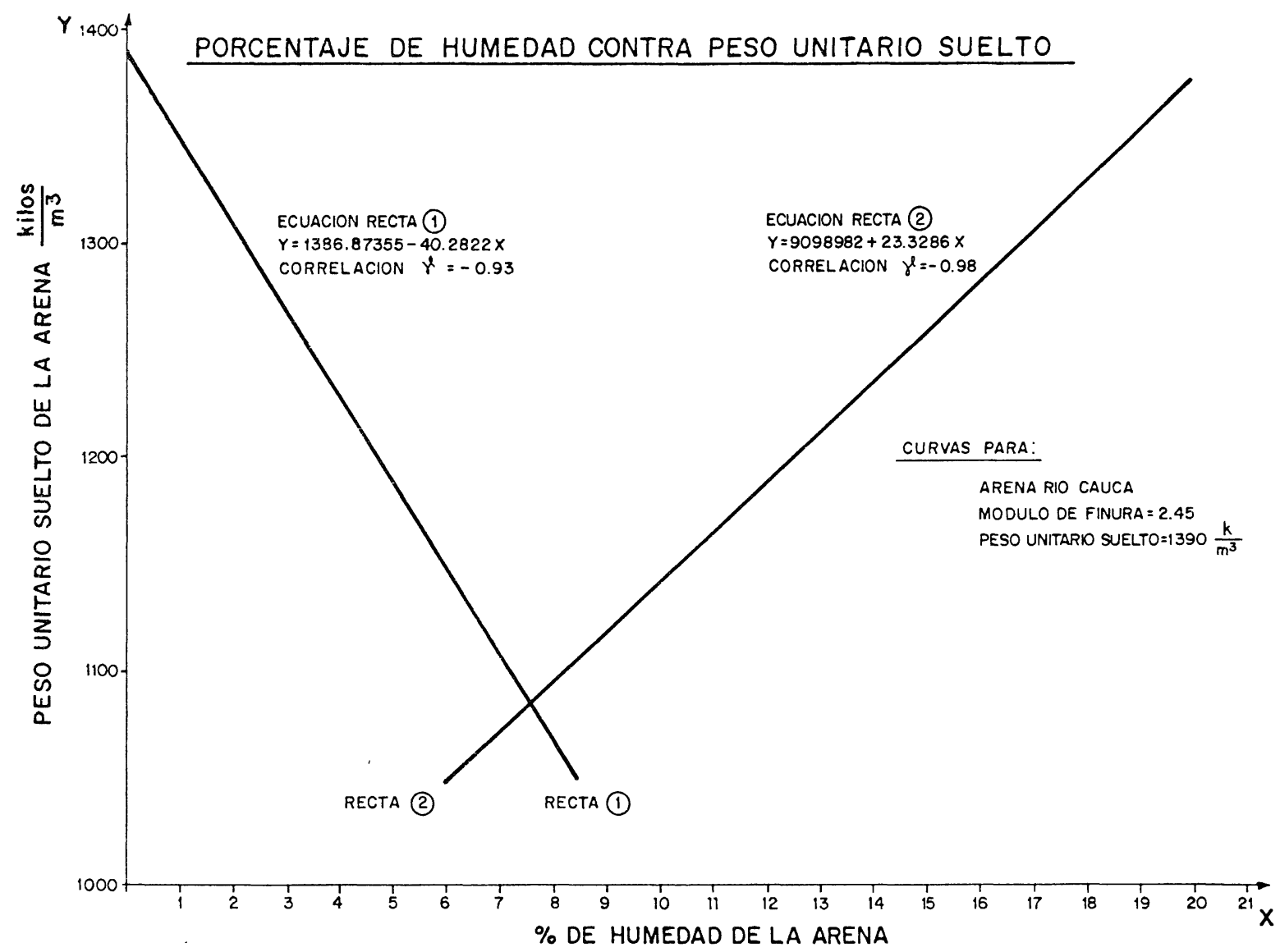

Gráfico 2

Lo anterior tiene gran importancia en la dosificación de morteros y hormigones por volumen, práctica ampliamente utilizada en nuestro medio, pues al cambiar la humedad de la arena -ello ocurre frecuentemente dependiendo de la época del año- la medida de la cantidad de ésta por peso estará variando aunque se llene el mismo volúmen de material y con ella la trabajabilidad del hormigón y/o el mortero. Ante esta circunstancia se acude a adicionar más agua a la mezcla afectando las propiedades mecánico-resistentes negativamente.

\subsection{Variación del grado de hinchamiento de las arenas del Río Cauca, en función del contenido de humedad}

De idéntica forma que en 4.1. se procesó la información pertinente, obteniéndose las siguientes correlaciones:

a) En el rango de humedad entre 0.0 y $8.9 \%$ :

$$
\begin{aligned}
& \mathrm{Y}=3.71 \mathrm{X}-2,32(3) \\
& \mathrm{r}=0,97
\end{aligned}
$$


b) En el rango de humedad entre 8.9 y $26.0 \%$ :

$$
\begin{aligned}
& \mathrm{Y}=40.82-1.20 \times(4) \\
& \mathrm{r}=0.94
\end{aligned}
$$

Para ambos casos:

$$
\begin{aligned}
& \mathrm{X}=\% \text { de humedad de la arena } \\
& \mathrm{Y}=\% \text { de hinchamiento }
\end{aligned}
$$

En el gráfico 3 se observa que existe un máximo de hinchamiento del orden del $28.5 \%$ para un contenido de humedad del $8.9 \%$. Esta información es valiosa tanto para efectuar correcciones de las dosificaciones de mezcla por volumen como para evitar "pérdidas de inventarios" en la compra y acopio de arenas.

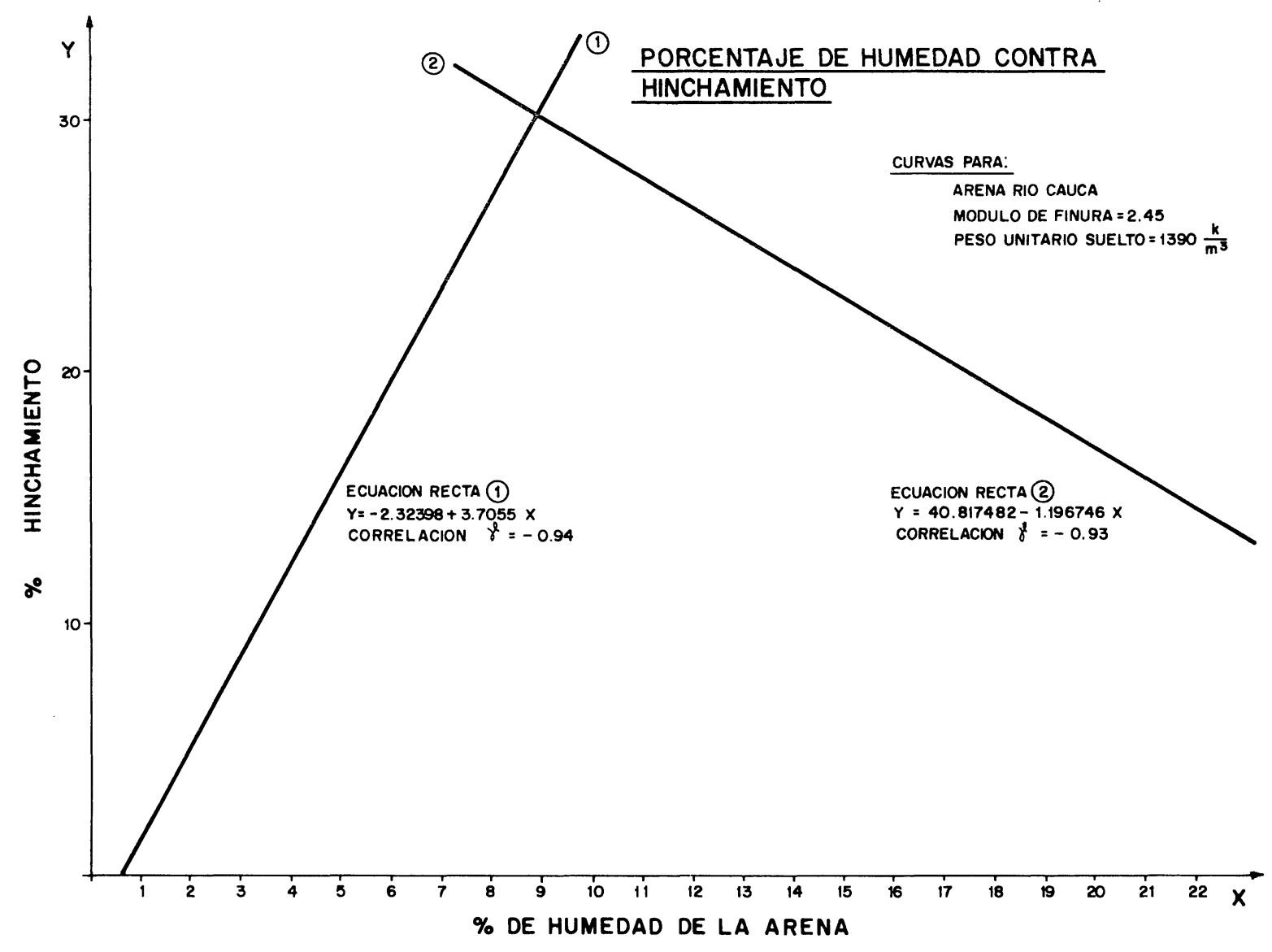

Gráfico 3

En forma indirecta, se puede conseguir el grado de humedad de una arena del Río Cauca apta para mezclas, si se determina su hinchamiento. Asi se podrá corregir adecuadamente el proporcionamiento de una mezcla (9).

Es importante anotar que el efecto de la humedad sobre el hinchamiento y el peso unitario, sólo comienza cuando se supera el estado de superficie seca saturada. Recuérdese que el hinchamiento es un fenómeno ocasionado por la humedad superficial en los granos de arena. 


\subsection{Correlaciones entre las relaciones arena/cemento $y$ agua/cemento $y$ las resistencias mecánicas a 28 días}

4.3.1. Correlación de la relación arena/cemento y la resistencia mecánica a compresión a 28 días, para un rango de fluidez dado.

El procesamiento estadístico planteó una ecuación de la forma: (Ver gráfico 4).

$$
Y=\frac{K_{1}}{K_{2}^{\mathrm{Ar} / \mathrm{c}}}
$$

donde:

$$
\begin{aligned}
& K_{1}=689,87 \\
& K_{2}=1,434
\end{aligned}
$$

quedando así:

$$
f_{\mathrm{c}}=\frac{689,87}{(1.434)^{\mathrm{Ar} / \mathrm{c}}}
$$

Coeficiente de Correlación $(r):-0.94$.

$\mathrm{Ar} / \mathrm{c}=$ relación arena/cemento entre 2 y 5 .

$f_{\mathrm{c}}=$ resistencia a la compresión a 28 días $\left(\mathrm{kg} / \mathrm{cm}^{2}\right)$.

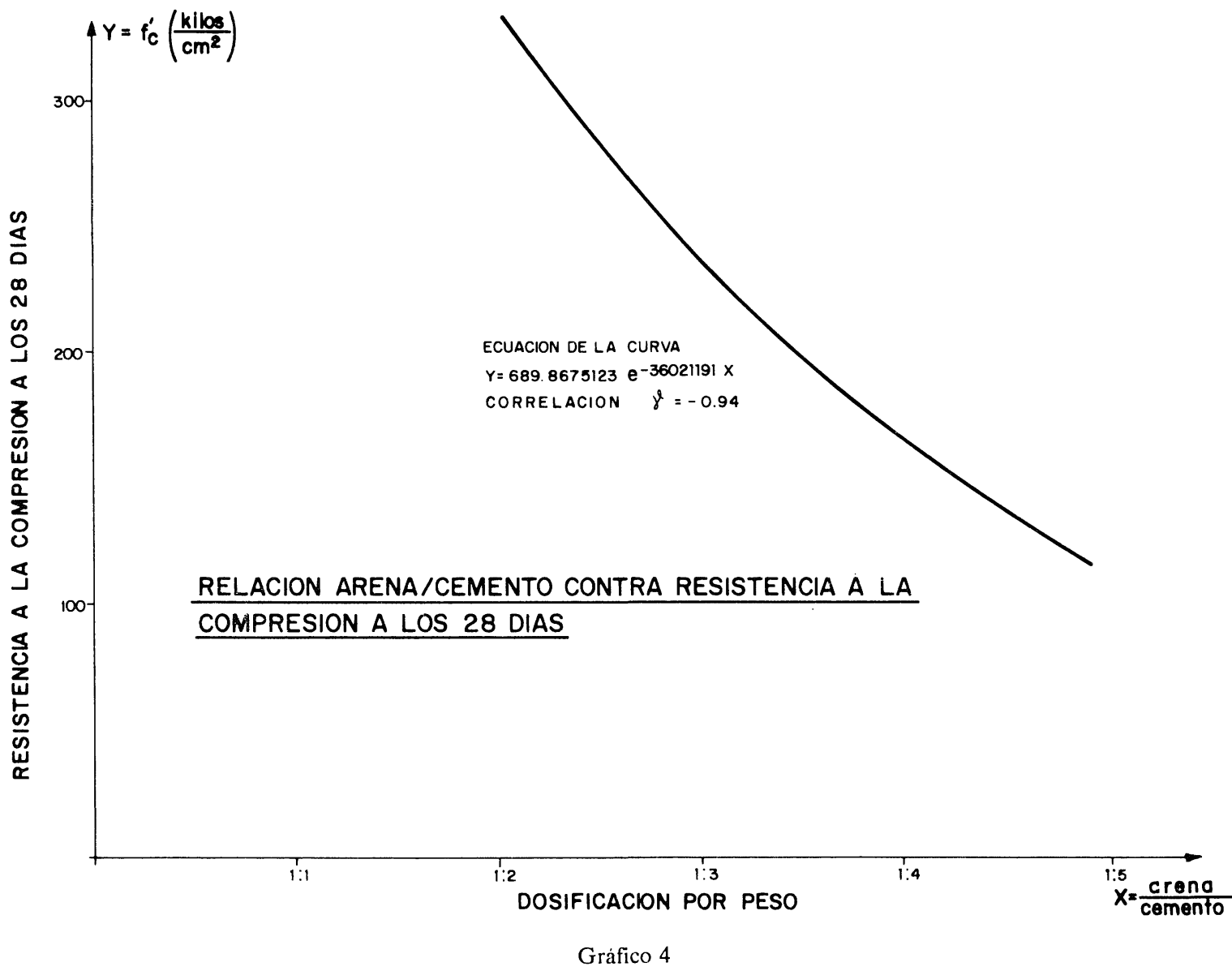

MATERIALES DE CONSTRUCCION. Vol. 35, n. ${ }^{\circ} 197$, enero/febrero/marzo 1985 


\section{Condiciones de la ecuación}

- Arena Rio Cauca. Módulo de finura $=2.56$.

- Cemento Conquistador tipo portland.

- Agua del Acueducto de Cali.

- Fluidez media del mortero $=103 \%$.

$$
\sigma=5.6 \% \text { (dosificación estándar). }
$$

C.V. $=5.4 \%$ (coeficiente de variación).

4.3.2. Correlación de la relación agua/cemento y la resistencia mecánica a compresión a 28 días, para un rango de fluidez dado.

El procesamiento estadistico planteó una ecuación de la forma: (Ver gráfico 5)

$$
Y=\frac{K_{1}}{K_{2}^{\text {a/c }}}
$$

donde:

$$
\begin{aligned}
& K_{1}=678,68 . \\
& K_{2}=6,4475 .
\end{aligned}
$$

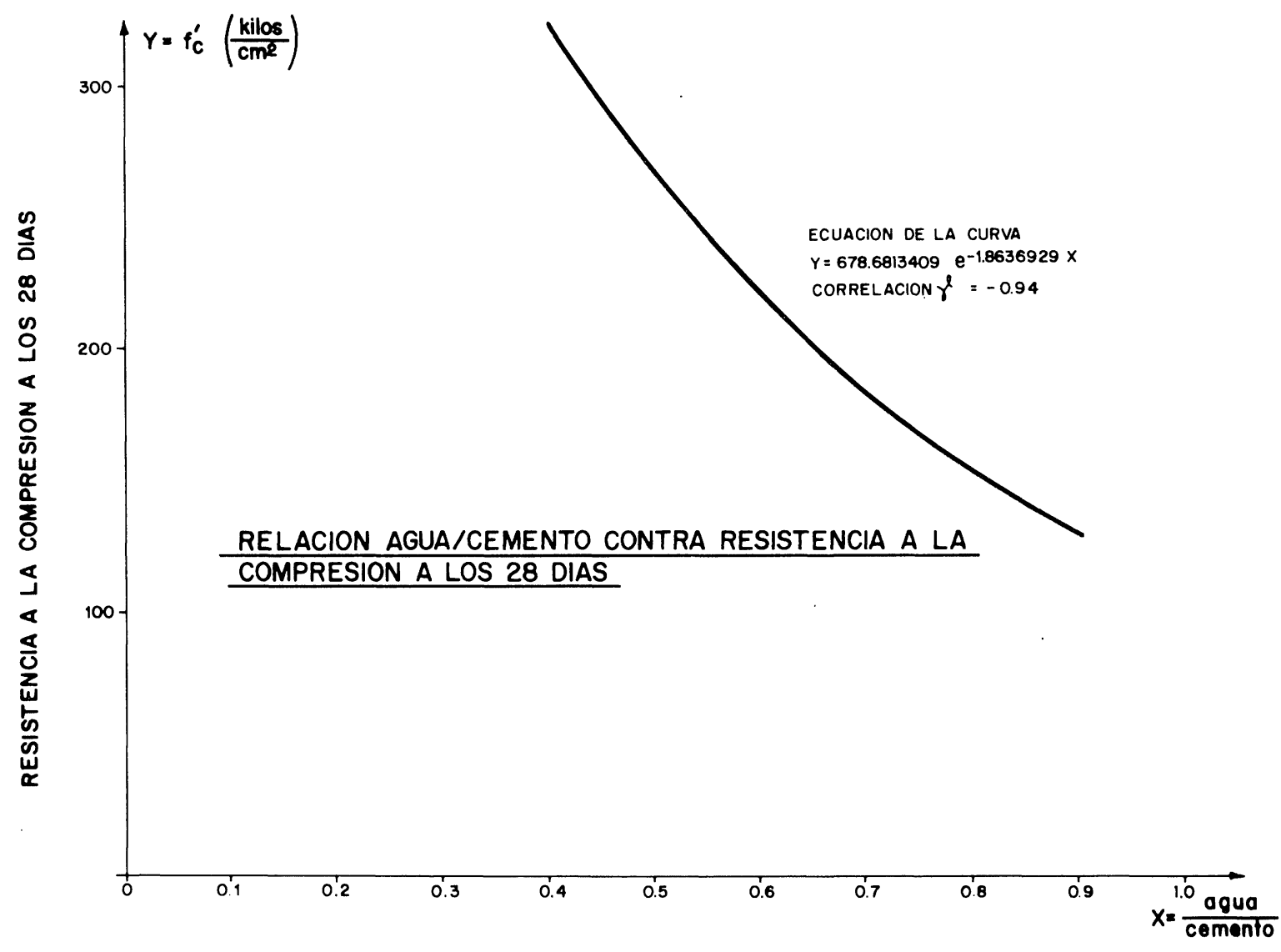

Gráfico 5 
quedando asi:

$$
f_{\mathrm{c}}=\frac{678,68}{(6.4475)^{\mathrm{a} / \mathrm{c}}}
$$

Coeficiente de Correlación $(r)=-0.94$

donde:

$\mathrm{a} / \mathrm{c}=$ relación agua/cemento entre 0.40 y 0.90 .

$f_{\mathrm{c}}=$ resistencia a la compresion a 28 días $\left(\mathrm{kg} / \mathrm{cm}^{2}\right)$.

Condiciones: La ecuación se aplica para las mismas condiciones indicadas en 4.3.1.

\subsection{Evaluación del Peso Volumétrico del Mortero}

\subsubsection{Peso Volumétrico Suelto del Mortero Fresco}

Se procesaron estadísticamente los datos obtenidos, consiguiéndose los siguientes resultados:

- Peso Volumétrico Suelto promedio $=2140.7 \mathrm{~kg} / \mathrm{m}^{3}$

- Desviación Estándard = $\quad 55.56 \mathrm{~kg} / \mathrm{m}^{3}$

- Coeficiente de Variación $\quad=\quad 2.60 \%$

- Número de datos $\quad=\quad 37$

Se concluye que para morteros elaborados con arena del Río Cauca media el peso volumétrico suelto del mortero fresco, cuya fluidez ésta entre 95-110\%; es independiente de: la dosificación, la relación agua/cemento, la humedad de la arena. En términos generales se comporta como una constante.

\subsubsection{Peso Volumétrico compactado del Mortero Fresco}

Después de un procesamiento estadístico de los datos reportados, se obtuvieron los siguientes resultados:

- Peso Volumétrico Compactado promedio $=2277.3 \mathrm{~kg} / \mathrm{m}^{3}$

- Desviación Estándard

- Coeficiente de Variación

- Número de datos

$$
\begin{aligned}
& =\quad 97.24 \mathrm{~kg} / \mathrm{m}^{3} \\
& =\quad 4.27 \% \\
& =\quad 21
\end{aligned}
$$

Se determina que el peso volumétrico compactado de un mortero cuyas características sean similares a las descritas en 4.4.2. es una constante.

Lo anterior constituye el punto fundamental para plantear un método de diseño de mezcla de morteros.

Así mismo, estos resultados permiten determinar el rendimiento del mortero fresco recién mezclado con el colocado; esto se determina por la relación entre los pesos volumétricos suelto y compactado. El rendimiento del mortero, para las caracteristicas especificas, está alrededor del $94 \%$. 


\subsection{Análisis y Evaluación del Proceso de Retemplado}

El análisis se basa en determinar el efecto que tiene sobre la resistencia mecánica del mortero a 28 días la adición de agua de retemplado, hasta ubicarse en el rango de fluidez definido de 95-110\%. Se tomó como patrón de referencia la resistencia obtenida por la mezcla original sin adición de agua de retemplado.

Como el mortero permanece quieto e inconfinado, al remezclarlo pasado unos tiempos relativamente largos $(45,90,135 \mathrm{~min})$, se puede pensar en que se efectúa así una nueva dispersión del cemento cuando éste ha comenzado a reaccionar con el agua con la consiguiente rotura de la estructura de enlaces generada hasta el momento.

Se observa que a medida que transcurre el tiempo hay un decrecimiento de las resistencias. El efecto no es significativo en los primeros 45 minutos, tiempo al cual todavía no ha fraguado. La fluidez alcanzó un valor promedio del $95 \%$, cuando habían transcurrido 90 minutos del amasado. (Ver gráfico 6).

Se concluye que el retemplado perturba las resistencias finales del mortero. (Ver gráfico 7). Podrian existir dos explicaciones a estos comportamientos:

a) A los 90 minutos el cemento ha iniciado el desarrollo de las reacciones de hidratación e incluso ha empezado a densificarse el Gel del Cemento, a esto se debe la pérdida de plasticidad que este cemento presenta a los 75 minutos (Ver item 3.2.); así, al romper por mezclado y dilución dicho entumecimiento, la mezcla pierde el desarrollo potencial de resistencias que se habían dado hasta el momento.

b) Por adición del agua se perturba la relación agua/cemento original y con ello las resistencias obtenidas después del retemplado.

Este último fenómeno es el que mayor incidencia puede tener en las pérdidas de resistencia, pues la fluidez antes del retemplado era en promedio de $90 \%$ cuando el límite inferior aceptable es del $95 \%$ y ello confiere aún cierta plasticidad a la mezcla lo que indica que el fenómeno descrito en (a) aún no es muy significativo.

En algunos momentos o circunstancias, a pie de obra, se pueden combinar ambos casos.

Al efectuarse el retemplado de la mezcla original a los 135 min el efecto es mayor pues hay una pérdida de resistencia del orden del $17 \%$. (Ver gráfico 8).

La explicación del fenómeno es similar a la dada anteriormente.

En general se puede concluir que una mezcla de mortero podrá permanecer durante 45 minutos

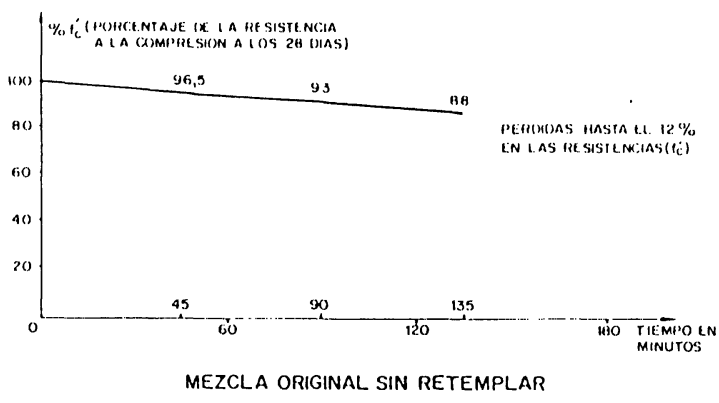

Gráfico 6

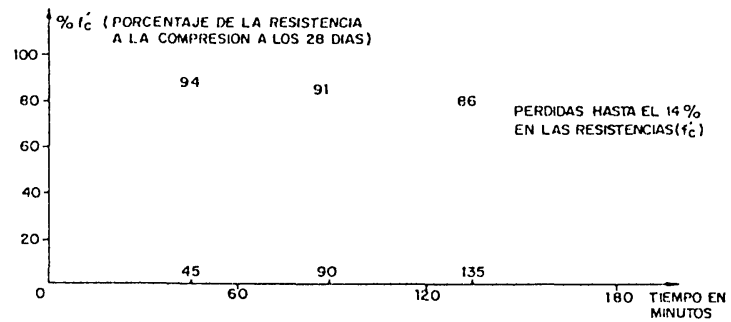

MEZCLA ORIGINAL RETEMPLADA A LOS 9OA 135 MIN.

Gráfico 7 
con una plasticidad y fluidez dentro del rango específico y su resistencia no se verá afectada significativamente; pasado este tiempo, con o sin adición de agua, el hecho de remezclar generará una caída de resistencias y a mayor edad será más notoria, así mismo la adición de agua incide en una mayor caida de la resistencia final.

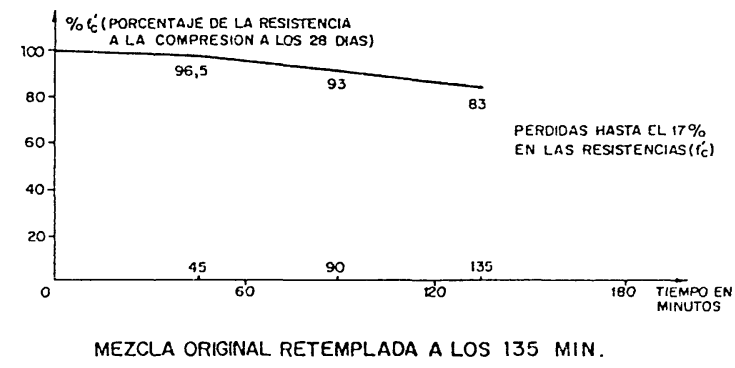

\subsection{Porcentaje de Humedad del Mortero fresco}

Gráfico 8

Al procesar la información de los porcentajes de humedad total de los morteros frescos correspondientes a las mezclas con un rango de fluidez entre 95 y $110 \%$, se encontró:

- \% de humedad media del mortero fresco $=16.14 \%$

- Desviación Estándar $=0.99 \%$

- Coeficiente de Variación $=6.14 \%$

- Número de datos $=42$

Todo para las condiciones de los materiales especificados anteriormente.

Se concluye que todo mortero, que satisfaga las caracteristicas referidas en esta investigación, tendrá un contenido de humedad total constante para conseguir una trabajabilidad óptima. Si se posee un medidor rápido de humedad, se podrá establecer un control de calidad aceptable y agil para los morteros producidos.

\subsection{Método de Diseño de Mezclas para dosificación por peso}

Consiste en aplicar los siguientes pesos, extraidos de las conclusiones anteriores:

4.7.1. Planteamiento del sistema de ecuaciones:

$\begin{array}{ll}\text { Ecuación (5): } & f_{\mathrm{c}}^{\prime}=\frac{689.87}{(1.434)^{\mathrm{Ar} / \mathrm{c}}} \\ \text { Ecuación (6): } & f_{\mathrm{c}}^{\prime}=\frac{678.68}{(6.4475)^{\mathrm{a} / \mathrm{c}}}\end{array}$

Ecuación (7): Peso volumétrico suelto $=\mathrm{Ar}+\mathrm{c}+\mathrm{a}$

$2140.7 \mathrm{~kg}=\mathrm{Ar}+\mathrm{c}+\mathrm{a}$

donde:

$f_{\mathrm{c}}=$ resistencia a la compresión a 28 días.

$\mathrm{Ar}=$ cantidad de Arena $(\mathrm{kg})$.

c $\quad=$ cantidad de Cemento $(\mathrm{kg})$.

$\mathrm{Ar} / \mathrm{c}=$ relación Arena/Cemento.

a $=$ cantidad de agua $(\mathrm{kg})$. 
4.7.2. La solución del sistema de ecuaciones planteado define las cantidades de materiales aptos para conseguir un mortero de fluidez definida y una resistencia a la compresion requerida. La arena en estas expresiones corresponde a un material en superficie Seca Saturada. Habrá necesidad de realizar las correcciones correspondientes debidas a la humedad de la arena cuando se vaya a aplicar en obra.

\section{RECOMENDACIONES}

\subsection{La reproducibilidad del presente método de diseño de mezclas de morteros estará sujeto a:}

5.1.1. Que se desarrolle en un ambiente donde las condiciones sean controlables.

5.1.2. Que las mediciones de materiales sean correctas y por peso (arena, cemento, agua).

5.1.3. Que se haga un mezclado mecánico de los morteros, a velocidades y tiempos establecidos por las Normas ICONTEC.

Por las razones expuestas se considera que la aplicación del método debe ir acompañado de un Factor de Seguridad, dependiendo de las condiciones del área y formas de trabajo. El valor del factor de seguridad debe ceñirse a lo especificado en este aspecto para el diseño de mezclas de hormigón, en cuanto a la resistencia característica de diseño (10), (11).

Como recomendaciones generales se dan:

- Para condiciones poco controlables y con un equipo de medición deficiente, se incrementa la resistencia de diseño por un Factor de Seguridad de 1.3.

- Para condiciones controlables y con equipo de medición y mezclado aceptable, se incrementa la resistencia de diseño por un Factor de Seguridad de 1.15.

La resistencia de diseño por el factor de seguridad determina la resistencia aplicable al método de Cálculo de la Mezcla.

\subsection{Recomendaciones debido a la humedad de la Arena}

5.2.1. La obtención de las cantidades por peso, al aplicar el método de diseño de mezclas para una resistencia establecida, sólo exige el conocimiento del porcentaje de humedad de la arena.

Por lo tanto, se recomienda medir la humedad de la arena antes de comenzar cada tajo de trabajo o en el transcurso de éste si existen notables cambios.

5.2.2. En lo que respecta a la trabajabilidad del Mortero, en aquellas dosificaciones donde el porcentaje de humedad de la arena es menor que el porcentaje de absorción, el agua adicional correspondiente a la corrección por humedad no es rápidamente absorbida por la arena, quedando una porción de agua libre que incrementa notablemente el porcentaje de fluidez; entonces, como recomendación para el diseño de mezclas de mortero, el porcentaje de humedad de la arena debe ser superior al porcentaje de absorción de la misma.

\subsection{Dosificación por Volumen}

Conocidas las cantidades por peso de Cemento, Arena y Agua corregidas, se pasan a dosificaciones por volumen como se indica a continuación. 
5.3.1. El peso del agua en kilos $=$ volumen de agua en litros.

5.3.2. El peso del cemento en kilos, dividido entre el peso unitario del cemento, es igual al volumen del cemento:

$$
\text { Volumen del Cemento }\left(\mathrm{m}^{3}\right)=\frac{\text { Peso del cemento (kilos) }}{\text { Peso unitario del cemento }\left(\mathrm{kg} / \mathrm{m}^{3}\right)}
$$

El peso unitario del cemento se encuentra entre 900 y $1.300 \mathrm{~kg} / \mathrm{m}^{3}$.

5.3.3. El Volumen de la Arena es igual a:

$$
\text { Volumen de la Arena }\left(\mathrm{m}^{3}\right)=\frac{\text { Peso de la arena (kilos) } \times(1+\% \text { Hinchamiento })}{\text { Peso unitario suelto de la arena seca } \mathrm{kg} / \mathrm{m}^{3}}
$$

Donde: El Peso Unitario Suelto y el hinchamiento son funciones del porcentaje de humedad de la arena. Se recomienda que para determinar el volumen de la arena es necesario conocer el comportamiento del peso unitario y del hinchamiento respecto al porcentaje de humedad de la arena con que se vaya a trabajar.

\section{BIBLIOGRAFIA}

(1) 1975 Annual Book of ASTM Standards. Part. 13. ASTM 1975.

(2) A. SAlAZAR: "Manual de Diseño de Mezclas de Concreto". En publicación.

(3) ICPC. Memorial “1. ${ }^{\circ}$ Curso sobre construcción en muros estructurales”. Octubre 1981.

(4) HECTOR RESTREPO: "Evolución de los Sistemas Constructivos en Colombia en la última década". ICPC Memorial 1. ${ }^{\circ}$ Curso sobre Construcción en Muros Estructurales. 1981.

(5) ICONTEC: Norma Técnica C 4.99/77.

(6) WILliAM GALlEGO, H.: "Mortero de Pega y mortero de Relleno para Mamposteria Estructural" ICPC. Memorias $1 .^{\circ}$ Curso sobre Construcción en muros estructurales. 1981.

(7) WILliAM GALlEGO, H.: "El bloque estructural en la Construcción actual”. Tesis de grado. Universidad Nacional de Colombia. Medellin 1980.

(8) A. SÁlAZAR: "Diseño de mezclas de morteros de pega. Un método empírico". Informe final de Investigación. Universidad del Valle. Facultad de Ingenieria. Sept. 1984.

(9) A. SALAZAR: "Tecnologia de Agregados y Agua". Universidad del Valle - Continuar - Mayo 1984.

(10) A. SALAZAR: "Sintesis de la Tecnologia del Concreto 2. parte". Universidad del Valle - Continuar - Julio 1984.

(11) A.C.I.-704: "Control de Calidad del Concreto". IMCYC/8. 1974. 